Microscopical examination of the scrapings from the hand revealed fungi, but unfortunately no attempt at culture was made. When the crusts had been removed several kerion lesions the size of a shilling were seen and a diagnosis of "cattle ringworm" was made, although there was no apparent source of infection. The kerion phase settled down, but was followed by the appearance of yellowish perifollicular crusts found to contain masses of fungi. The picture was that of favus, but there was no fluorescence under Wood's light. Culture at this stage produced a colony which was identified as $T$. violaceum. In view of this finding $x$-ray epilation was carried out, and appears to have been completely successful. Inquiry into a possible source of infection brought to light an Indian school friend, who was persuaded to attend for examination. She has subsequently been proved to be suffering from $T$. violaceum infection (Case 2) and was the apparent cause of infection.

Case 2.-A 10-year-old Indian girl had come to this country six years previously. On examination all that was found were five or six small scars, and a little fine scaling over the vertex of the scalp. There were no broken hairs, no crusts, and no fluorescence under Wood's light. The scaling, however, was in definite patches and was not quite typical of dandruff. Microscopical examination of scales and hairs on three ocrasions failed to show any fungi. but eventually one of numerous cultures made produced a typical colony of $T$. violoceum. Mother, father, and baby brother were examined without any abnormality of the scalp being discovered. The question of treatment is still undecided at the time of writing because of various racial problems.

Comment.-T. violaceum infections of the scalp apparently present themselves in very varied clinical appearances. The Indian girl showed lesions of superficial scaling of a degree which might have passed unnoticed. whilst the British girl developed favus and kerion-like lesions. I think it can be assumed that the British girl was infected by the Indian, and it seems probable, in view of the fact that other members of the Indian family were not infected, that she herself was infected in India.

I would like to thank Dr. Jaqueline Walker, of the Mycological Reference Laboratory, for her work on the identification of the cultures.

IAN ANDERSON, M.R.C.P., Consultant Dermatologist, Leicester Royal Infirmary. REFERENCES

Berlin, C., and Meyrovitz, C. (1955). Brit. J. Derm., 67, 397.

Franks. A. G., anj Taschdjian, C. L. (1956). A.M.A. Arch. Derm., 74. 349.

Lehmann. C. F., Pipkin. J. L., and Ressmann, A. C. (1950). Ibid., 61, 488

Miedzirski. F.. and Lipski. J., (1956). Brit. J. Derm., 68, 200.

Sagher. F. (1947). Art med. orient. (Tel-Aviv), 6, 68.

Walker, J. (1950). Brtt. J. Derm., 62, 239.

Whittle, C. H. (1956). Ibid., 68, 1.

\section{Foreign Body in the Hand}

The importance of the retained foreign body as a cause of chronic inflammation of the hand has been stressed by many authors. Bunnell (1944) gives prominence to such lesions; Lake (1955) describes the case of a patient who presented with a rubber ring embedded in granulations at the base of a finger.

The following case illustrates the reaction of living tissue to the presence of a foreign body, and the nature of the foreign body renders it of unusual interest.

\section{CASE RePort}

The patient, a married woman of 74 , first attended hospital in June, 1955. At that time she had noticed the development of swelling of the left ring-finger distal to her gold wedding-ring, which she admits was " rather tight." The ring was removed, and it was noted that there was an abrasion of the skin surface beneath the ring. Following removal of the ring the swelling of the finger subsided and the skin healed-taking four weeks to do so, however.

In October, 1955, she returned to hospital with inflammation of the affected finger and a thin purulent discharge at the site of the previous abrasion, on the dorsum of the proximal phalanx. The lesion failed to heal with conservative treatment, and culture of the pus yielded a growth of Staph. pvogenes sensitive to penicillin and oxytetracycline. No antibiotic had been given previously, and a course of systemic penicillin was therefore begun. $X$-ray examination of the digit showed some osteoarthritic changes in the metacarpo-phalangeal joint, but no evidence of bony erosion or of a radio-opaque foreign body.

The finger continued to discharge intermittently, and when seen in January, 1956, there was slight swelling over the proximal phalanx, with superficial granulations on the dorsum. surmounting a groove at the level of the proximal skin crease of the fin-

ger. A s mall amount of $\mathrm{th}$ i purulent discharge could be expressed at one point on the lateral aspect. It was decided to explore the finger, and this was done on January 23.

Under general anaesthesia th e superficial granulations were excised, and on deepening the incision through the groove a foreign body was identified about $\frac{1}{8}$ in. ( 3 $\mathrm{mm}$.) below the surface. The incision was continued around the finger and an intact elastic band was ex-

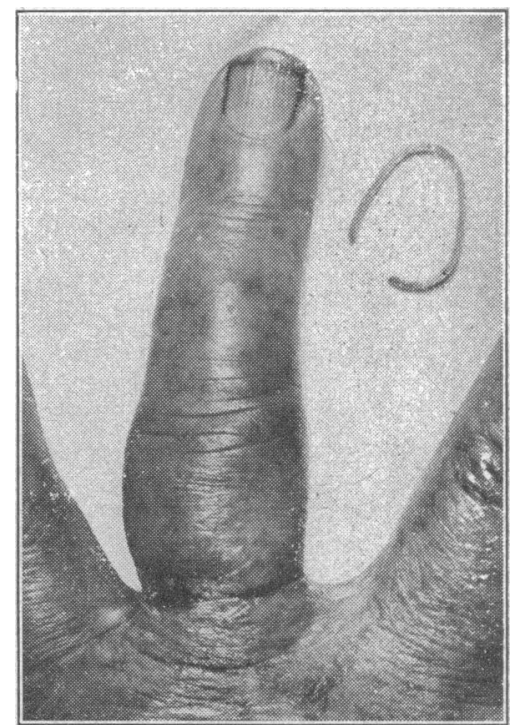

State of tinger one day after operation showing the removed elastic band. posed below the skin surface. There was no granulation tissue on the palmar aspect of the digit. The band was removed and the remaining granulations were curetted. Two weeks from the date of operation healing was complete and there was no swelling of the finger.

The accompanying photograph shows the state of the finger one day after operation, together with the elastic band.

\section{COMMENT}

Upon further questioning the patient supplied the information that at one time her work involved the handling of small elastic bands, used for securing small parcels. She had been in the habit of slipping the bands on to her fingers. It is presumed that the band in question had lain beneath the wedding-ring for a considerable period of time, becoming embedded by a process of pressure necrosis. It is interesting that the area subjected to pressure necrosis by the elastic band was not apparent at the time that the wedding-ring was removed from the finger, the only evidence of trauma then being the small abrasion on the dorsum of the finger. The elastic band had become completely submerged beneath the skin surface, and healing around the circumference of the finger, superficial to the foreign body, was almost complete.

We thank Mr. J. B. Hume for permission to publish this case. The department of medical photography at St. Bartholomew's Hospital was responsible for taking the photograph.

$$
\begin{aligned}
& \text { J. I. BURN, M.B., F.R.C.S., } \\
& \text { Late registrar. } \\
& \text { P. H. N. WooD, M.B., B.S., } \\
& \text { Late house-surgeon. } \\
& \text { Casualty Department, St. Bartholomew's Hospital, London. }
\end{aligned}
$$

REFERENCES

Bunnell, S. (1944). Surgery of the Hand, p. 474. Lippincott, Philadelphia. Lake, N. C. (1955), Brit med. J. 2, 1550 . 